\title{
Physical-statistical channel model for signal effect by moving human bodies
}

\author{
Michael Cheffena
}

\begin{abstract}
A novel physical-statistical channel model for simulating the signal effect by moving human bodies is presented. The human body is modeled as vertically oriented dielectric cylindrical volume. The received signal is assumed to be composed of a direct component which might be subject to shadowing and a multipath component due to reflection and diffuse scattering, i.e., a Ricean channel. The shadowing effect of the direct signal component is calculated using Kirchhoff diffraction equation. The multipath component is parameterized by calculating the reflected fields from the floor, ceiling and walls of the indoor environment as well as scattered fields from moving human bodies. Poisson and Exponential distributions are used to describe the shadowing and inter-shadowing events caused by multiple bodies, respectively. Furthermore, simulation results of the first and second order statistics of the received signal affected by moving human bodies for $3.35 \mathrm{GHz}$ and $60 \mathrm{GHz}$ signals are presented. In addition, initial validation of the developed model are performed using an empirical model for human body shadowing and reported measurement results.
\end{abstract}

Keywords: channel model, indoor propagation, fading, human body

\section{Introduction}

Wireless communication systems are subject to different propagation impairments, among them are signal shadowing by moving human bodies. For wireless systems operating in indoor environments, human body shadowing is a significant propagation impairment. Unlike to outdoor cellular systems, the transmission power and the height of base-stations is much lower in indoor environments. Thus, the signal loss by body shadowing greatly affects the received signal strength. Furthermore, giving a fixed location of the transmitting and receiving antennas, the random movement of people may cause a time varying channel conditions.

A path-shadowing model for indoor environment using Markov process is reported in [1]. In [2,3], numerical examination of electromagnetic wave shadowing by human body using method-of-moment (MoM) solutions are presented. A two-state Ricean model for predicting the indoor wireless channel was reported in [4]. An empirical model for describing the large-scale fading pattern of the signal affected by the human body was presented in [5]. Furthermore, reference [6] presents

Correspondence: Michael.Cheffena@hig.no

Gjøvik University College, Teknologivn. 22, Gjøvik N-2815, Norway propagation measurement results in the presence of human activity for a $60 \mathrm{GHz}$ channel. In [7], the effect of human body shadowing on the multiple-input-multiple-output (MIMO) channel performance were investigated.

In this article, we propose a physical-statistical channel model for signal effect by moving human bodies. Physical-statistical models are more effective for simulating large scenarios compared to complex physical models, and more accurate than merely empirical or statistical models as they take into account the geometry of the link, and rely on physical (electromagnetic based) methods for calculating the needed model parameters.

The received signal is assumed to be composed of a direct component which might be subject to shadowing and a multipath component due to diffuse scattering, i. e., a Ricean channel. The direct signal component is subject to shadowing effects, and is calculated using Kirchhoff diffraction equation. The multipath component is parameterized by calculating the reflected fields from the floor, ceiling and walls of the indoor environment as well as scattered fields from moving human bodies which are modeled as finite dielectric cylinders. The parameters of the Rice distribution change as the 
people represented in terms of simple volumetric forms walks through the propagation environment. Furthermore, Poisson and Exponential distributions are used to describe the shadowing and inter-shadowing events caused by multiple bodies, respectively.

The article begins in Section 2 by identifying the main propagation mechanisms for moving human body effects in indoor environment, and presents the developed physical-statistical channel model. Simulation results and discussions are given in Section 3. Initial validation of the proposed model using an empirical model for human body shadowing and reported measurement results are shown in Section 4. Finally, conclusions are presented in Section 5.

\section{Physical-statistical channel model}

The considered propagation scenario is shown in Figure 1. The indoor propagation environment is defined by orthonormal vectors $\mathbf{x}, \mathbf{y}$, and $\mathbf{z}$ of a rectangular coordinate system. The human body is modeled as a finite lossy dielectric cylinder and is walking in the direction
$A B C$ or CBA crossing the line-of-sight (LOS) path between the transmitter $(\mathrm{Tx})$ and the receiver $(\mathrm{Rx})$ at position $\mathrm{B}$. The positions of the transmitting and receiving antennas are fixed.

In addition to the reflection of the signal by the floor, ceiling and walls of the indoor environment (reflections from furniture and other objects are not considered), the signal is also scattered by moving human body at, e.g., position $A$ and $C$ (see Figure 1). As the person approaches position $\mathrm{B}$, the signal is subject to both scattering and diffraction from the top and side of the human body. Note that the strength of the scattered and diffracted signals are position dependent and change as the person walks though the propagation environment. Furthermore, the random movement of people create time varying channel conditions. Characterization of the Doppler spectra is thus important for the determination of the time variance of the wireless channel. Other important parameters are the shadowing and inter-shadowing intervals which are needed when simulating the crossing of the LOS path by multiple bodies.

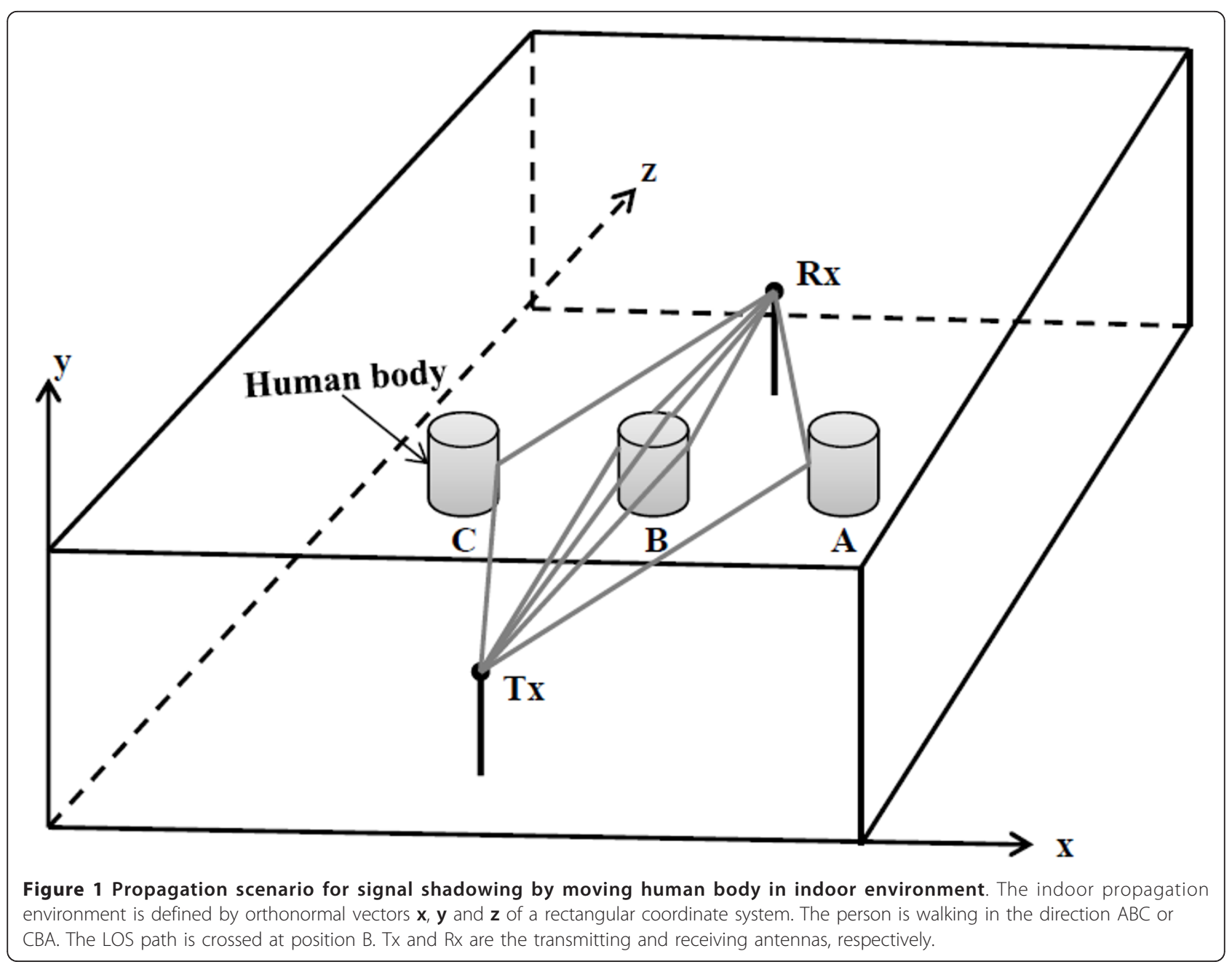


In subsequent sections, we discuss each of the aforementioned propagation mechanisms for signal effect by moving human bodies.

\subsection{Reflection and scattering}

The strength of the signal reflected by the floor, ceiling and walls of the indoor environment depend on the link configuration and material properties of the reflecting surfaces. The reflection coefficient is given by [8]

$$
\begin{aligned}
& R_{N}=\frac{\cos \theta_{r}-\sqrt{\eta-\sin ^{2} \theta_{r}}}{\cos \theta_{r}+\sqrt{\eta-\sin ^{2} \theta_{r}}} \\
& R_{P}=\frac{\cos \theta_{r}-\sqrt{\left(\eta-\sin ^{2} \theta_{r}\right) / \eta^{2}}}{\cos \theta_{r}+\sqrt{\left(\eta-\sin ^{2} \theta_{r}\right) / \eta^{2}}}
\end{aligned}
$$

where $R_{N}$ and $R_{P}$ are the reflection coefficients when the electric field component is normal and parallel to the reflection plane, respectively. Parameter $\theta_{r}$ is the angle between the incident ray and the normal to the reflecting surface. Parameter $\eta$ is the complex permittivity of the reflecting material. The received reflected signal power can then be calculated using

$$
P=\frac{P_{t} G_{t} G_{r}}{L_{p} L_{r}}
$$

where $P_{t}$ is the transmitted power, $G_{t}$ and $G_{r}$ are transmitting and receiving antenna gains, respectively. Parameter $L_{p}$ is the path lose and $L_{r}=|R|^{2}$ is the reflection lose with $R=R_{P}, R_{N}$.

The signal is also scattered by people moving in the propagation environment. An illustration of the human body model adopted in this study is shown in Figure 2. The human body is modeled as a vertically oriented cylindrical volume in a rectangular coordinate systems defined by the orthonormal vectors $\mathbf{x}, \mathbf{y}$, and $\mathbf{z}$. The radios $R$ and the height $H$ describe the dimension of the volume. Incident on the human body is a linearly polarized wave with wavenumber $k=2 \pi / \lambda, \lambda$ being the wavelength, and direction of propagation $\mathbf{k}_{i}$. The transmitter is assumed to be located far away from the human body, so that the incident field amplitude can be approximated as being constant over the human body. Similarly, the scattered wave is received at a point located far away from the human body, in the direction $\mathbf{k}_{s}$ relative to the center of the human body. The propagation vectors of the incident $\mathbf{k}_{i}$ and scattered $\mathbf{k}_{s}$ fields are given by (see Figure 2)

$$
\mathbf{k}_{i}=-\sin \theta_{i} \cos \phi_{i} \mathbf{z}-\sin \theta_{i} \sin \phi_{i} \mathbf{x}-\cos \theta_{i} \mathbf{y}
$$

$$
\mathbf{k}_{s}=\sin \theta_{s} \cos \phi_{s} \mathbf{z}-\sin \theta_{s} \sin \phi_{s} \mathbf{x}-\cos \theta_{s} \mathbf{y}
$$

where angles $\theta_{i}$ and $\varphi_{i}$ are the elevation and azimuth directions of the incident field. Angles $\theta_{s}$ and $\varphi_{s}$ are the elevation and azimuth directions of the scattered field.

The scattering of an arbitrarily polarized wave incident on the cylinder can be described in terms of the scattering amplitude tensor, $F\left(\mathbf{k}_{s}, \mathbf{k}_{i}\right)$ [9]. If the field inside the cylinder is estimated as the field inside a similar, but infinite cylinder, then $F\left(\mathbf{k}_{s}, \mathbf{k}_{i}\right)$ can be expressed as in [9, Equation (47)]. The scattering cross-section can then be calculated using

$$
\sigma_{\mathrm{Cs}}=4 \pi\left|F\left(\mathbf{k}_{s}, \mathbf{k}_{i}\right)\right|^{2}
$$

For the propagation scenario shown in Figure 1, at each location of the human body, the incident and scattering angles can be calculated using geometrical relations, and these can be used further to determine the scattered signal utilizing the scattering cross-section defined in Equation (6).

The multipath (fast fading) component of the Rice channel in indoor environment might then be described by the sum of the standard deviations of the reflected and scattered fields, $\sigma_{\text {reflected }}+\sigma_{\text {scattered. }}$ Together with the direct signal component (discussed in Section 2.2), these are used in Section 2.5 to generate the signal fading caused by moving human body.

\subsection{Diffraction}

As the person approaches position B in Figure 1, the signal is also subject to diffraction from the top and side of the human body. The diffracted signal can be calculated using Kirchhoff diffraction equation [10,11] which gives the relationship between the aperture diffracted electric field at an observation point, $P$, and the freespace electric field

$$
\frac{E(P)}{E_{\mathrm{O}}(P)}=\frac{j}{2} \iint_{\Sigma(u, v)} \exp \left(-j \frac{\pi}{2}\left(u^{2}+v^{2}\right)\right) d u d v
$$

with

$$
\left[\begin{array}{l}
u \\
v
\end{array}\right]=\frac{\sqrt{2}}{R_{1}}\left[\begin{array}{l}
x_{\mathrm{o}} \\
y_{\mathrm{o}}
\end{array}\right]
$$

where $O$ is the intersection point (with Cartesian coordinates $x_{\mathrm{o}}$ and $y_{\mathrm{o}}$ ) of the Tx-Rx straight line with the obstacle (see Figure 3). Parameter $\Sigma(u, v)$ is the surface of the aperture, and $R_{1}$ is the radius of the first Fresnel zone given by

$$
R_{1}=\sqrt{\frac{\lambda a b}{a+b}}
$$




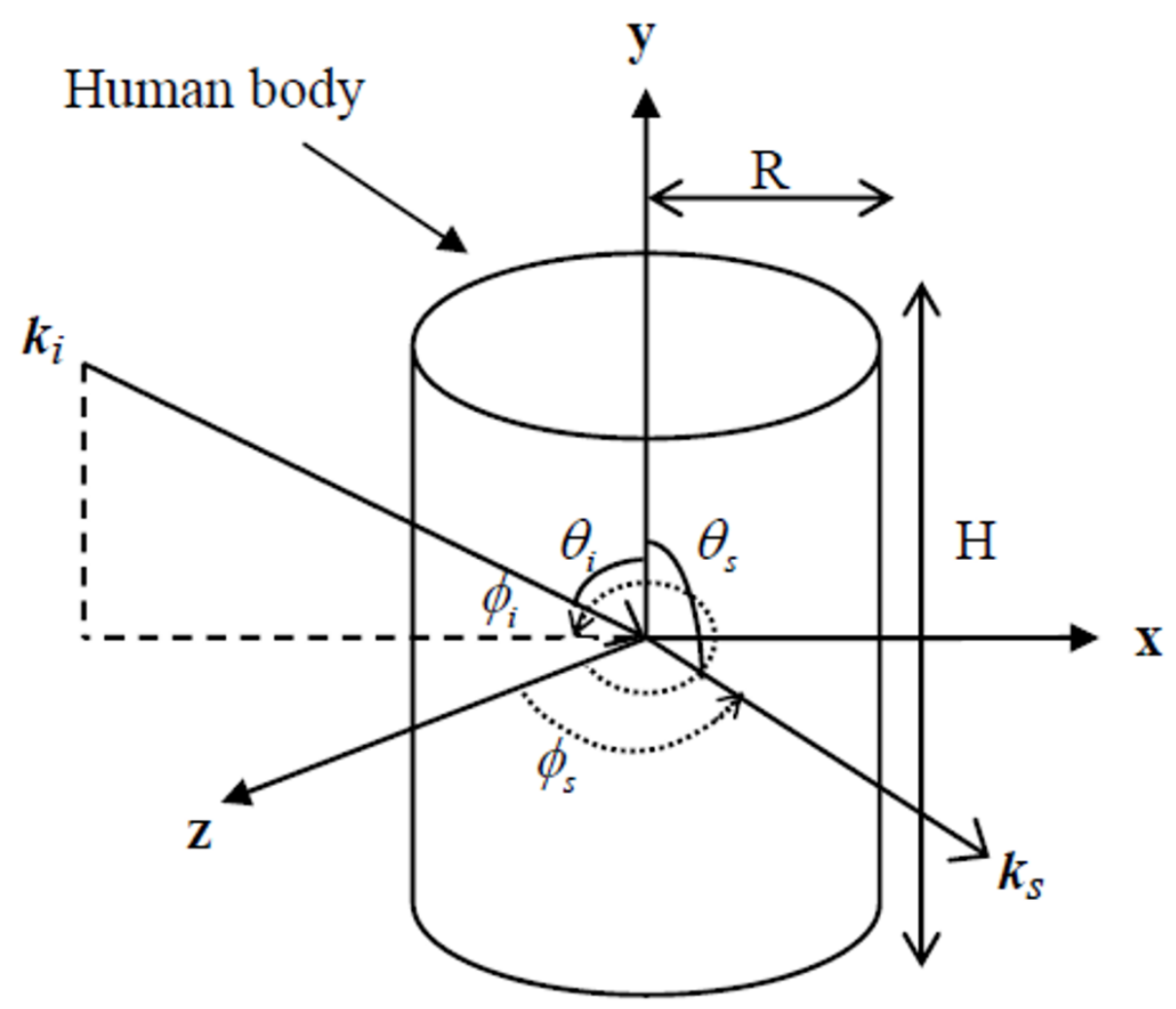

Figure $\mathbf{2}$ Human body model. $\mathbf{x}, \mathbf{y}$ and $\mathbf{z}$ are the orthonormal vectors of rectangular coordinate system. $R$ and $H$ are the radius and height of the human body. $\mathbf{k}_{i}$ and $\mathbf{k}_{s}$ are the incident and scattered propagation vectors. $\theta_{i}$ and $\varphi_{i}$ are the elevation and azimuth directions of the incident field. $\theta_{s}$ and $\varphi_{s}$ are the elevation and azimuth directions of the scattered field.

where $a$ and $b$ are the distances from the transmitter and the receiver to the aperture plane.

The total received diffracted field is then the combination of the three aperture diffracted fields whose surfaces are $\Sigma_{1}, \Sigma_{2}$, and $\Sigma_{3}$ with assumed infinite depth. Unlike simple ray tracing and Markov process models, the transitions between LOS and non-line-of-sight (NLOS) conditions are not sharp and thus more realistic regarding the empirical results. Using this model, good estimation of the shadow region behind a human body might be obtained. The standard deviation of the diffracted field, $\sigma_{\text {diffracted }}$, is used to describe the direct (slow fading) component of the received signal affected by moving human body. Together with the multipath signal component (discussed in Section 2.1), $\sigma_{\text {diffracted }}$ is used in Section 2.5 to generate the signal fading caused by moving human body.

\subsection{Doppler spectrum}

The random movement of people in the propagation environment create time varying channel conditions.
Characterization of the Doppler spectra is thus important for the determination of the time variance of the wireless channel. The situation where the antenna is moving in a random environment leads to the classical Jakes spectrum (with bathtub like shape) for scatters uniformly distributed in azimuth [12]. For the case where the antenna is stationary, moving scatterers in the channel such as vehicles (in outdoor environment) or persons (in indoor environment) will lead to a different Doppler spectrum which peaks at $0 \mathrm{~Hz}$ and falls off rapidly [13,14].

For electrically large scatterer such as the human body with scattering pattern peaking in the forward direction, the Doppler spectrum is given by [15]

$$
S_{n}(f)=(-1)^{n} P_{n-0.5}\left(\frac{f^{2}}{2}-1\right)
$$

where $P_{n-0.5}$ is the $n$th order Legendre function. In [15], a strong correlation was found between the shape of the scattering pattern and the Doppler spectrum. Based on this, a simplified model which mimics the 


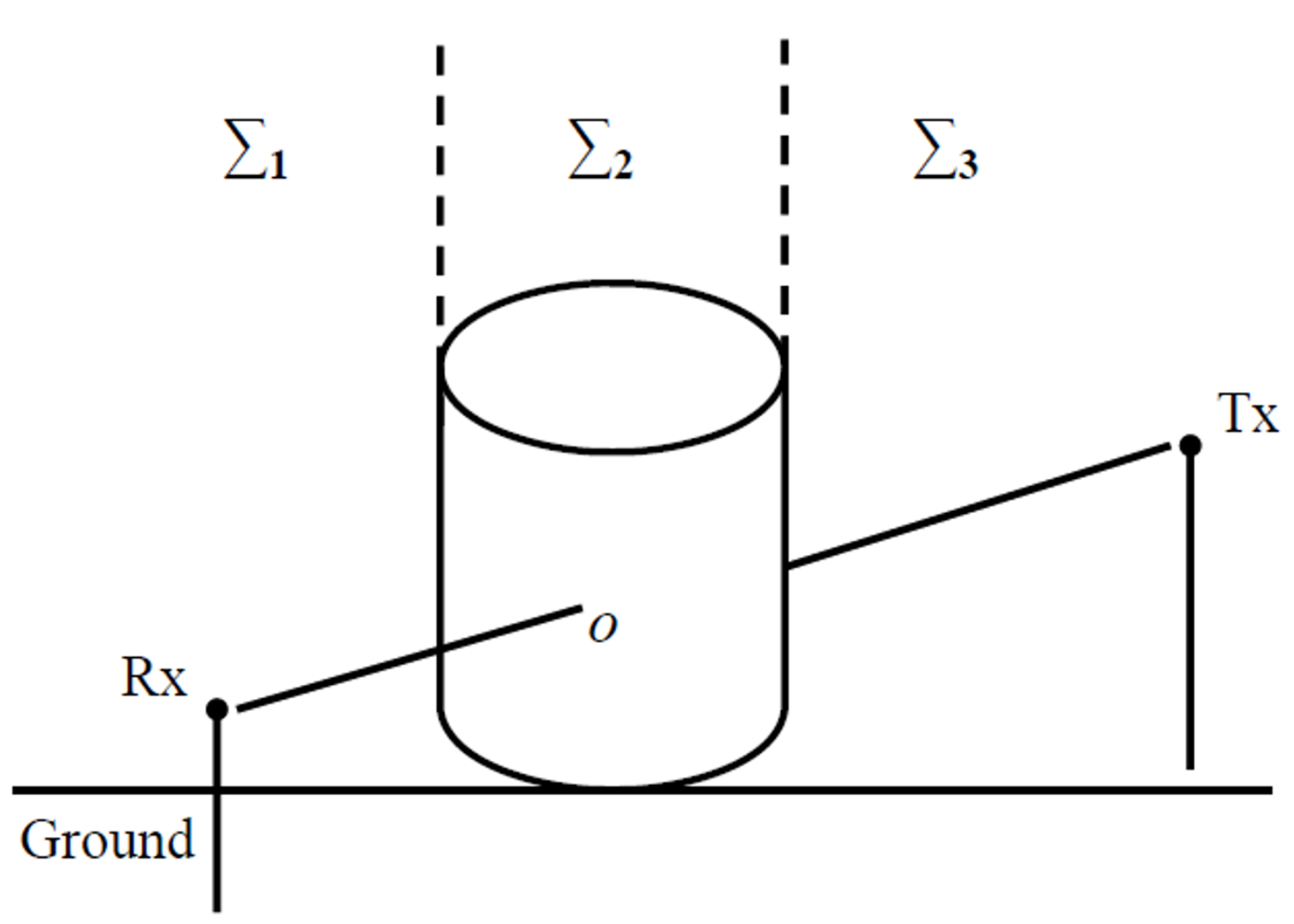

Figure 3 Model for signal diffraction by the human body. Parameters $\Sigma_{1}, \Sigma_{2}$, and $\Sigma_{3}$ are surfaces of the aperture. Parameter $O$ is the intersection point of the Tx-Rx straight line with the human body.

measured spectra caused by randomly moving people is proposed, and is given by [15]

$$
S(f)=\frac{1}{f+e}
$$

where $e$ is a model constant with value equal to 0.02 chosen to much the measured Doppler spectrum [15].

\subsection{Shadowing and inter-shadowing events}

Knowledge on the shadowing and inter-shadowing events are needed to simulate the multiple crossing of the LOS path by randomly moving people. Usually, an arrival process is described by Poisson distribution [16]. As in [1], the number of shadowing events may thus be modeled using Poisson distribution, expressed as

$$
p(N)=\frac{\widehat{N}^{N}}{N !} \exp (-\widehat{N})
$$

where $\widehat{N}$ is the average number of bodies crossing the LOS path. Equation (12) gives the probability that $N$ persons cross the LOS path within a given period of time.
In Poisson process, the Exponential distribution plays an important role in describing the inter-event durations [17]. For a Poisson process with parameter $\widehat{N}$, the time between events are Exponentially distributed, defined as

$$
p(T)=\widehat{N} \exp (-\widehat{N} T)
$$

where $T$ is the time interval to the next shadowing event. Equation (13) gives the probability of $T$ time interval for the next crossing of the LOS path. Parameter $\widehat{N}$ depends on the type of the environment, i.e., indoor (office, home, airport terminal, etc.) or outdoor (urban, suburban, rural, etc.), and should be characterized using measurements for the considered propagation environment.

\subsection{The simulation model}

The proposed physical-statistical channel model for simulating the signal effect by moving human body is shown in Figure 4. In the model, a complex white Gaussian processes with zero mean and unit variance is filtered by Equation (10) or Equation (11) for Doppler spectrum shaping. The resulting time series are 


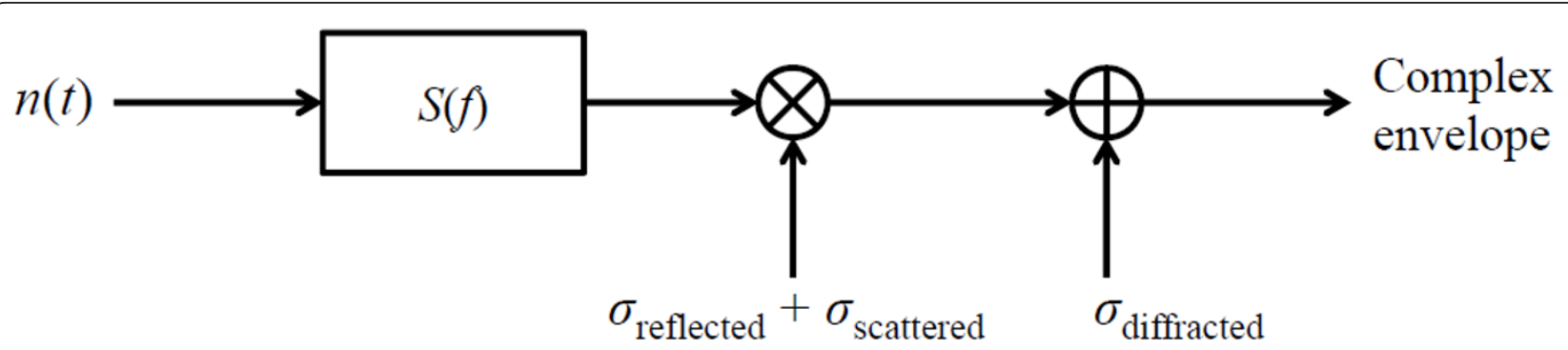

Figure 4 Channel simulator for the signal effect by moving human body. A complex white Gaussian processes with zero mean and unit variance is filtered by $S(f)$ (defined in Equation (10) or Equation (11)) for Doppler spectrum shaping. Parameters $\sigma_{\text {reflected, }} \sigma_{\text {scattered }}$ and $\sigma_{\text {diffracted }}$ are the standard deviations of the reflected, scattered and diffracted fields, respectively.

multiplied by the sum of the standard deviations of the reflected and scattered fields, $\sigma_{\text {reflected }}+\sigma_{\text {scattered }}$ (see Section 2.1) to produce the multipath (fast fading) component of the received signal. Then, the standard deviation of the diffracted field, $\sigma_{\text {diffracted }}$ (see Section 2.2) representing the direct (slow fading) signal component, is added to produce the total complex signal envelope of the received signal affected by moving human body.

This results in physical-statistical modeling of the propagation channel where the received signal envelope is Rice distributed with its position dependent parameters calculated using electromagnetic based methods (i.e., reflection, scattering, and diffraction). Note that parameters $\sigma_{\text {scattered }}$ and $\sigma_{\text {diffracted }}$ are position dependent, and change as the person walks through the propagation environment. Furthermore, Poisson and Exponential distributions are used to generate the shadowing and intershadowing events which can be used to simulate the crossing of the LOS component by multiple moving human bodies (see Section 2.4).

\section{Simulation results and discussions}

The scattering cross-section of a dielectric cylinder representing a human body for a vertically polarized signal at $3.35 \mathrm{GHz}$ and $60 \mathrm{GHz}$ is shown in Figure 5. Isotropic antennas are used at the transmitter and receiver. The simulation parameters are given in Table 1 . The field is incident from direction $\varphi_{i}=180^{\circ}$, so the forward scattering direction is $\varphi_{s}=0^{\circ}$. We can observe that the forward lobe of the scattering pattern becomes narrower with decreasing background as the frequency increases. This results in decreasing the received scattered fields in directions other than the forward direction.

An example of simulated time series at $3.35 \mathrm{GHz}$ and $60 \mathrm{GHz}$ using the developed physical-statistical channel model for multiple body crossing of the LOS path is shown in Figure 6. As expected we can observe that the signal attenuation by the human body increases with increasing frequency. We can also observe that the LOS path is crossed by three persons at around 5, 12, and 17 seconds with each shadowing event having a duration of around $1.5 \mathrm{~s}$. Note that the duration of each shadowing event depends on the walking speed and diameter of the human body, and my vary from person to person. Knowledge on the duration of shadowing events is important for designing fade mitigation techniques (FMTs) such as adaptive coding and modulation. Usually there is no prior knowledge of the walking speed, $v$, and radius, $R$, of the people crossing the LOS path in a given environment. One way is to use an average values of $v$ and $R$ as done in our simulations. A different approach might be to generate random values of $v$ and $R$ for each body crossing the LOS path from statistical distributions. In this case, statistical knowledge of $v$ and $R$ are required.

Figures 7, 8, and 9 show the simulated cumulative distribution functions (CDFs), level crossing rates (LCRs) and average fade durations (AFDs) of the received signal at $3.35 \mathrm{GHz}$ affected by multiple bodies $(N=1,2, \ldots, 5$ persons). The same is shown in Figures 10, 11, and 12 for $60 \mathrm{GHz}$ signal. Again we can observe that as the frequency increases the effect of moving human bodies on the first and second order statistics of the channel increases. Generally, as the frequency increases the wavelength decreases and thus small change of the path length may result in fast variations of the channel. From Figures 7 and 10 we can see that as the number of pedestrians increase (for $N=1,2, \ldots, 5$ ), the slope of the CDFs of the received signal decreases proportionally. Similarly, as the number of pedestrians increase, the LCRs and AFDs of the received signal increases, see Figures 8, 9, 11, and 12. These results are consistent with the measurement results of human body effects reported in [18].

\section{Model validation}

As discussed in Section 2, the multipath component results from summation of incoming waves reflected by the floor, ceiling and walls of the indoor environment as well as scattered by moving human bodies. While the 


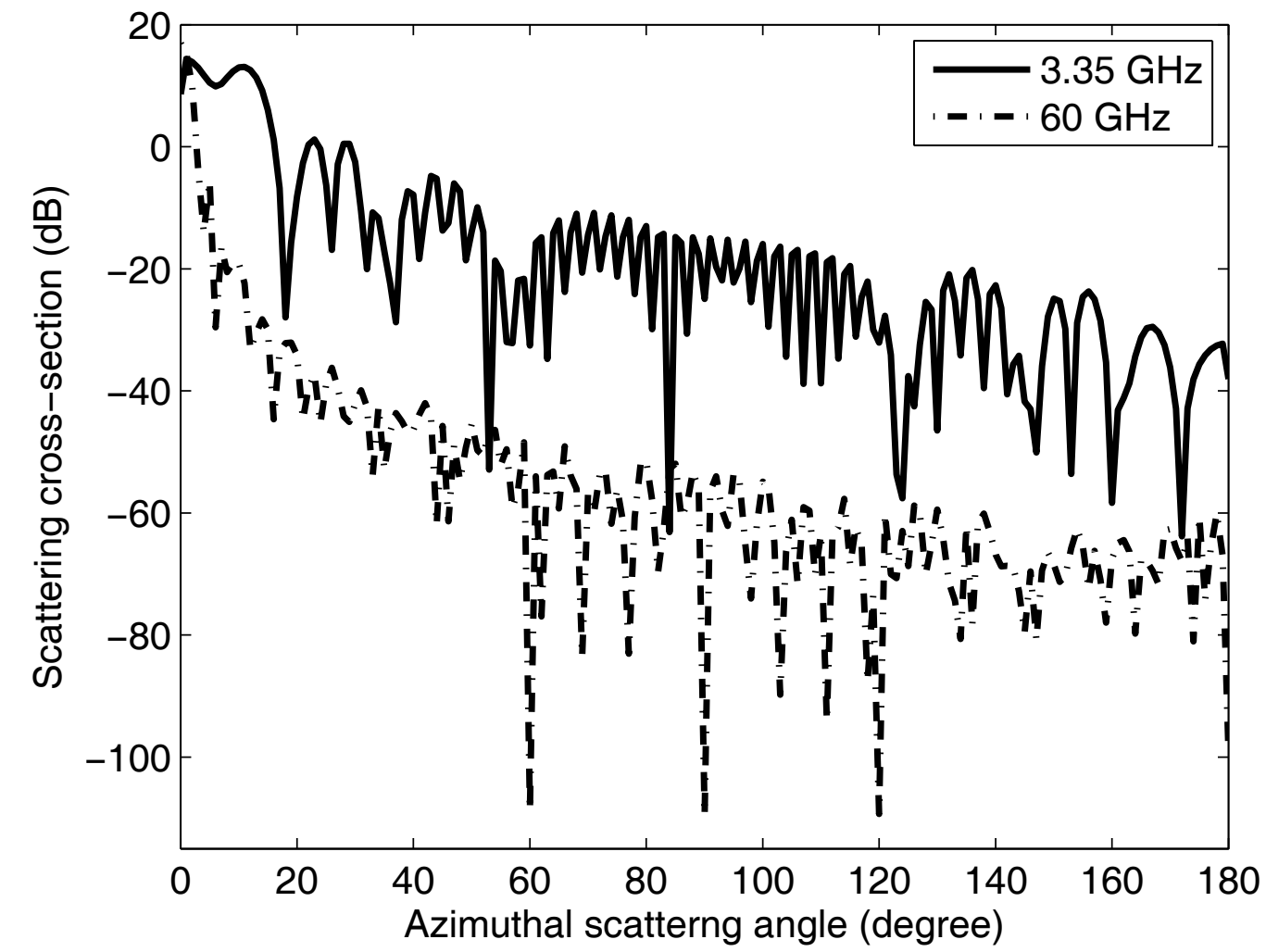

Figure 5 Scattering cross-section of a dielectric cylinder representing a human body at $3.35 \mathrm{GHz}$ and $60 \mathrm{GHz}$. The simulation parameters are given in Table 1.

direct signal component is mainly affected by obstructing people and is responsible for the large-scale fading observed on the received signal. Based on measurements, Pagani et al. [5] proposed a Gaussian-like empirical model for describing the large-scale fading pattern of the signal affected by the human by

Table 1 Simulation parameters

\begin{tabular}{ll}
\hline Parameter & Value \\
\hline Frequency, $f$ & $\begin{array}{l}3.35 \mathrm{GHz}, 60 \\
\mathrm{GHz}\end{array}$ \\
Tx antenna height, $h_{\mathrm{Tx}}$ & $1 \mathrm{~m}$ \\
$\mathrm{Rx}$ antenna height, $h_{\mathrm{Rx}}$ & $1 \mathrm{~m}$ \\
Average human body radius, $R$ & $0.18 \mathrm{~m}$ \\
Average human body height, $\mathrm{H}$ & $1.80 \mathrm{~m}$ \\
Average number of crossing, $\widehat{N}$ & 3 \\
Average walking speed, $v$ & $1 \mathrm{~m} / \mathrm{s}$ \\
Human body relative permittivity at $3.35 \mathrm{GHz}, \eta$ & $50-\mathrm{j} 100[19]$ \\
Human body relative permittivity at $60 \mathrm{GHz}, \eta$ & $11-\mathrm{j} 4.5[20]$ \\
Relative permittivity of the floor, ceiling and wall at & $5.31-\mathrm{j} 0.47[8]$ \\
$\begin{array}{l}3.35 \mathrm{GHz}, \eta \\
\text { Relative permittivity of the floor, ceiling and wall at } 60\end{array}$ & $5.31-\mathrm{j} 0.27[8]$ \\
$\mathrm{GHz}, \eta$ & \\
\hline
\end{tabular}

$$
\begin{gathered}
g(t)=-A_{s} \exp \left\{1-\left(\left(t-t_{0}\right) \frac{2}{T_{2}}\right)^{2}\right\} \\
\cdot \exp \left\{-u\left(\left(t-t_{0}\right) \frac{2}{T_{s}}\right)^{2}\right\}
\end{gathered}
$$

where $A_{s}$ represents the maximal shadowing attenuation in $\mathrm{dB}, T_{s}$ is the shadowing duration in seconds, $t_{0}$ is the shadowing instance, and $u$ is a model constant.

Figure 13 shows comparison of the direct signal component (large-scale fading) obtained using the empirical model given in Equation (14) and the one obtained using the proposed physical-statistical channel model at $3.35 \mathrm{GHz}$. Generally, we can observe that there is relatively good agreement between the two models. However, comparing to the empirical model given in Equation (14), the direct component of the physical-statistical channel model slightly oscillates due to the interference between the various unobstructed Fresnel zones. The same behavior can also be observed from measurement and numerical simulation (using MOM) results reported in [2]. In general, there is relatively high similarity between the measurement and numerical 


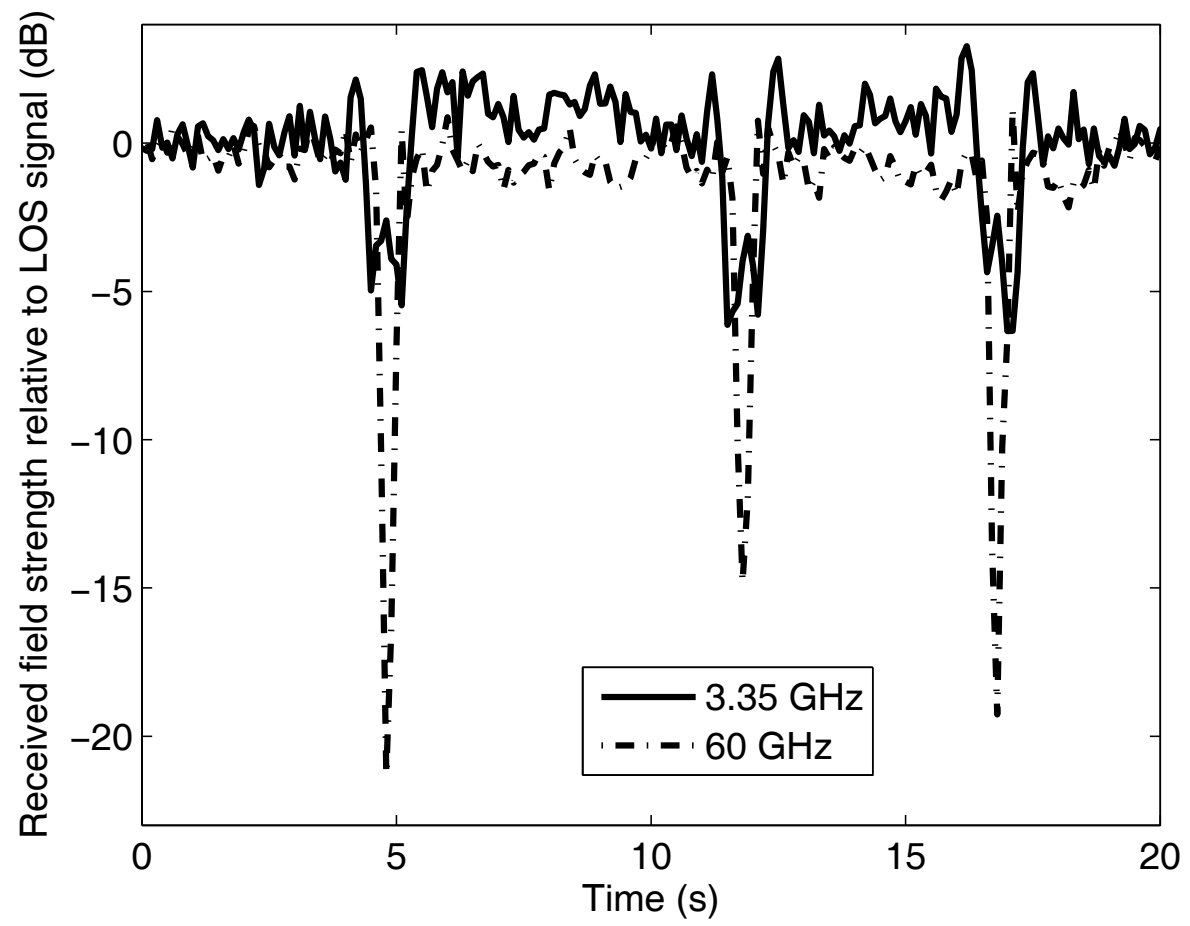

Figure 6 Simulated time series of the received signal effect by the human bodies at $3.35 \mathrm{GHz}$ and $60 \mathrm{GHz}$. The simulation parameters are given in Table 1.

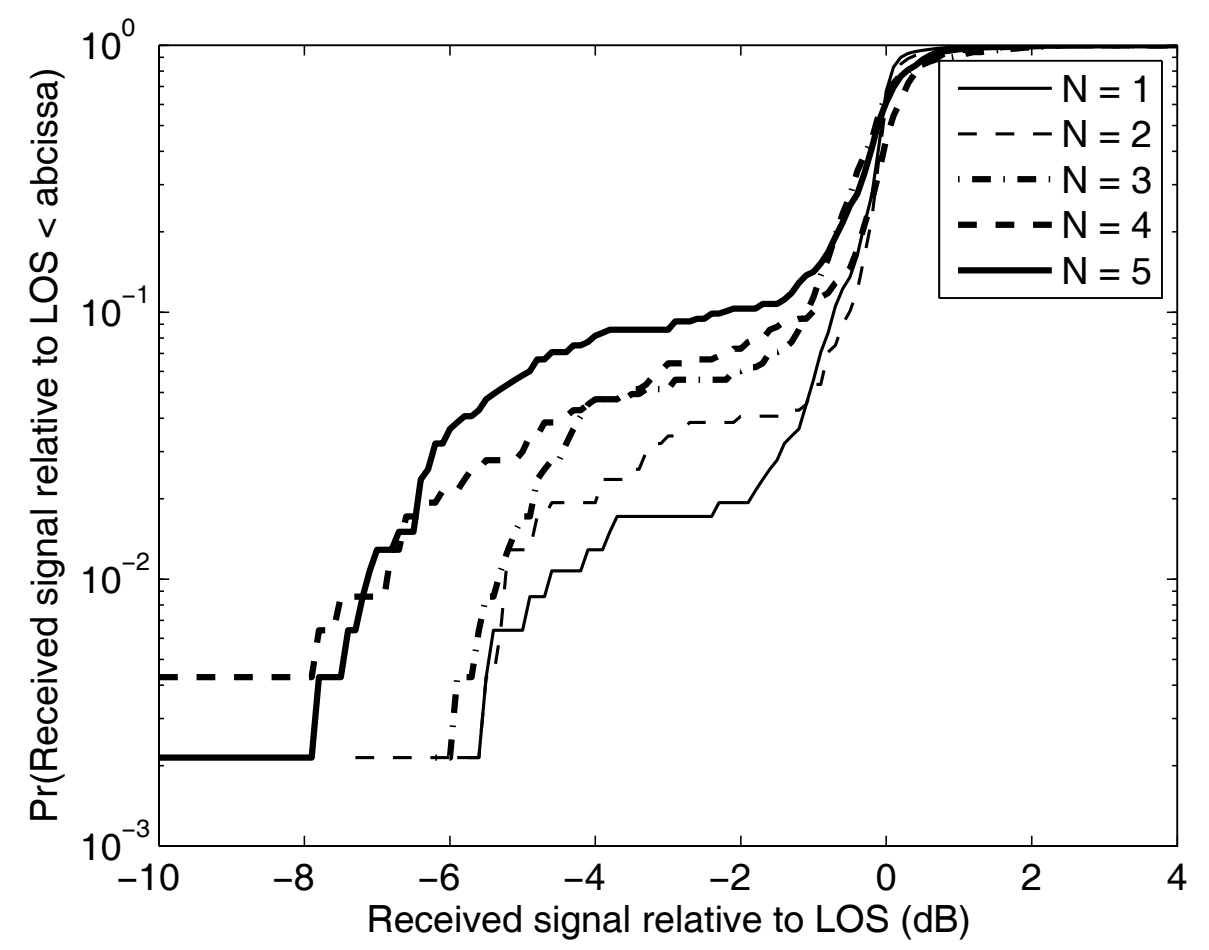

Figure 7 Simulated CDFs of the received signal effect by multiple human bodies $(N=1,2, \ldots, 5)$ at $3.35 \mathrm{GHz}$. The simulation parameters are given in Table 1. 


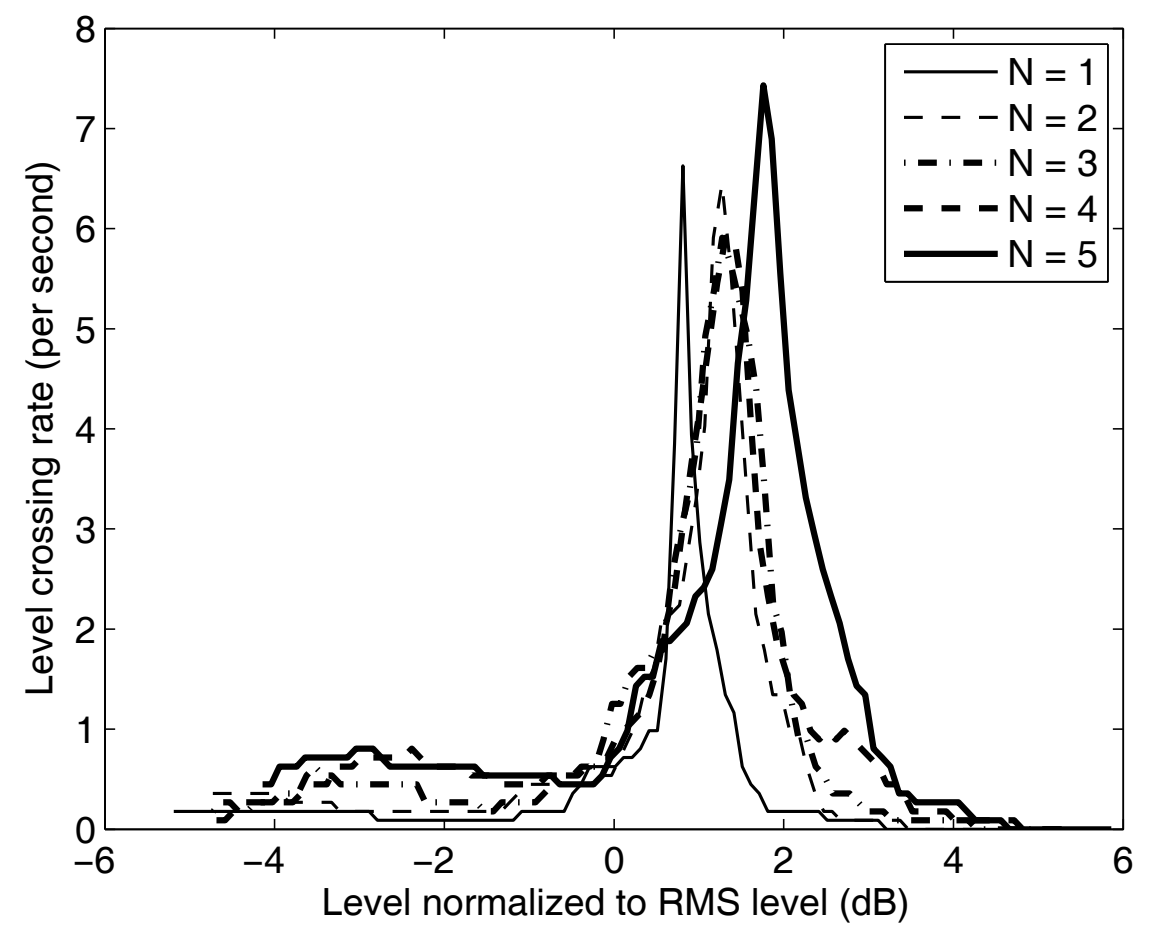

Figure 8 Simulated LCRs of the received signal effect by multiple human bodies $(\boldsymbol{N}=1,2, \ldots, 5)$ at $3.35 \mathrm{GHz}$. The simulation parameters are given in Table 1.

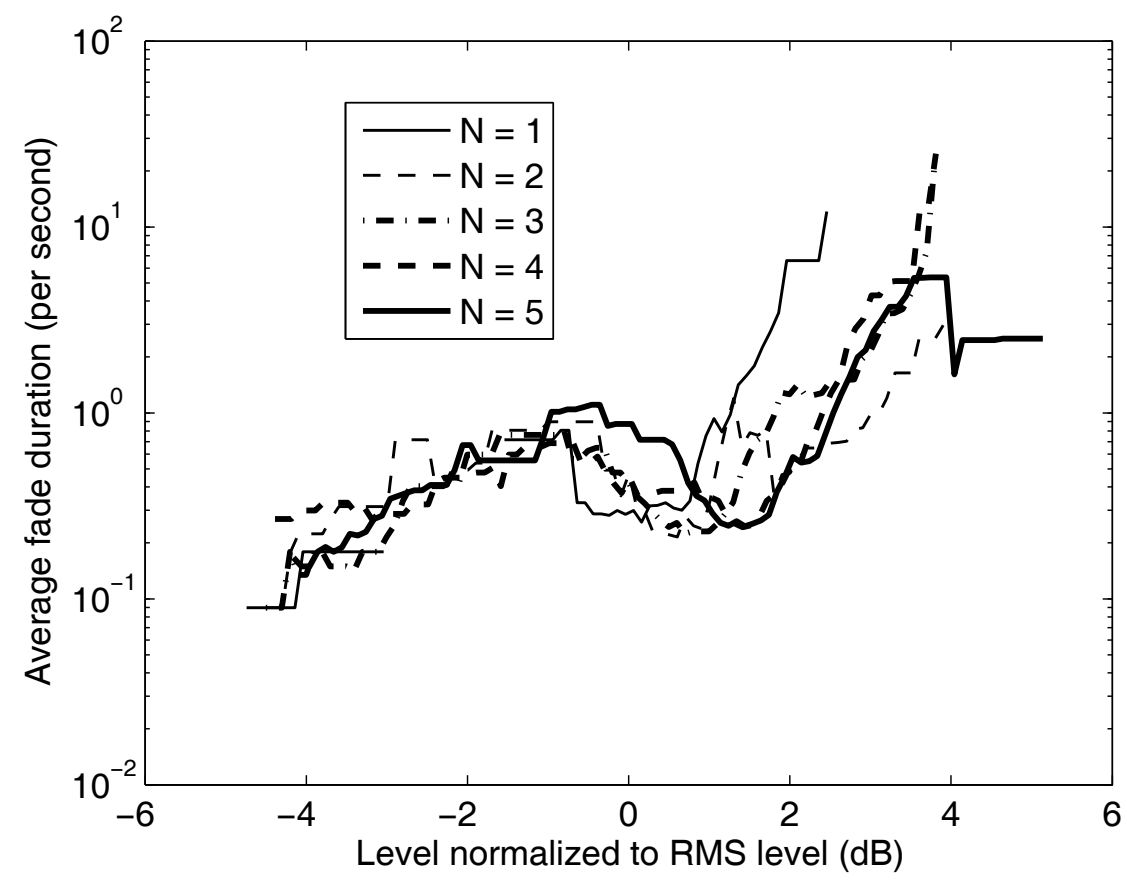

Figure 9 Simulated AFD of the received signal effect by multiple human bodies $(N=1,2, \ldots, 5)$ at $3.35 \mathrm{GHz}$. The simulation parameters are given in Table 1. 


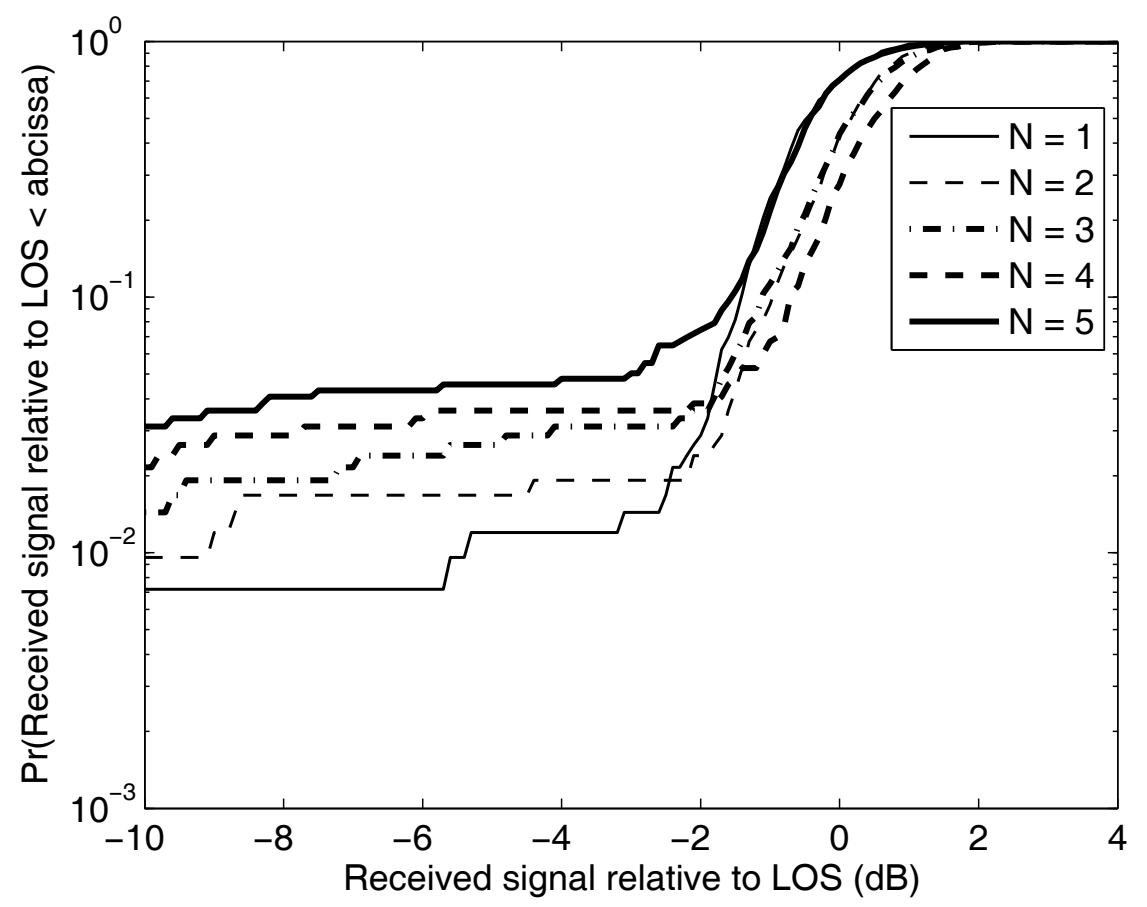

Figure 10 Simulated CDFs of the received signal effect by multiple human bodies $(N=1,2, \ldots, 5)$ at $60 \mathrm{GHz}$. The simulation parameters are given in Table 1.

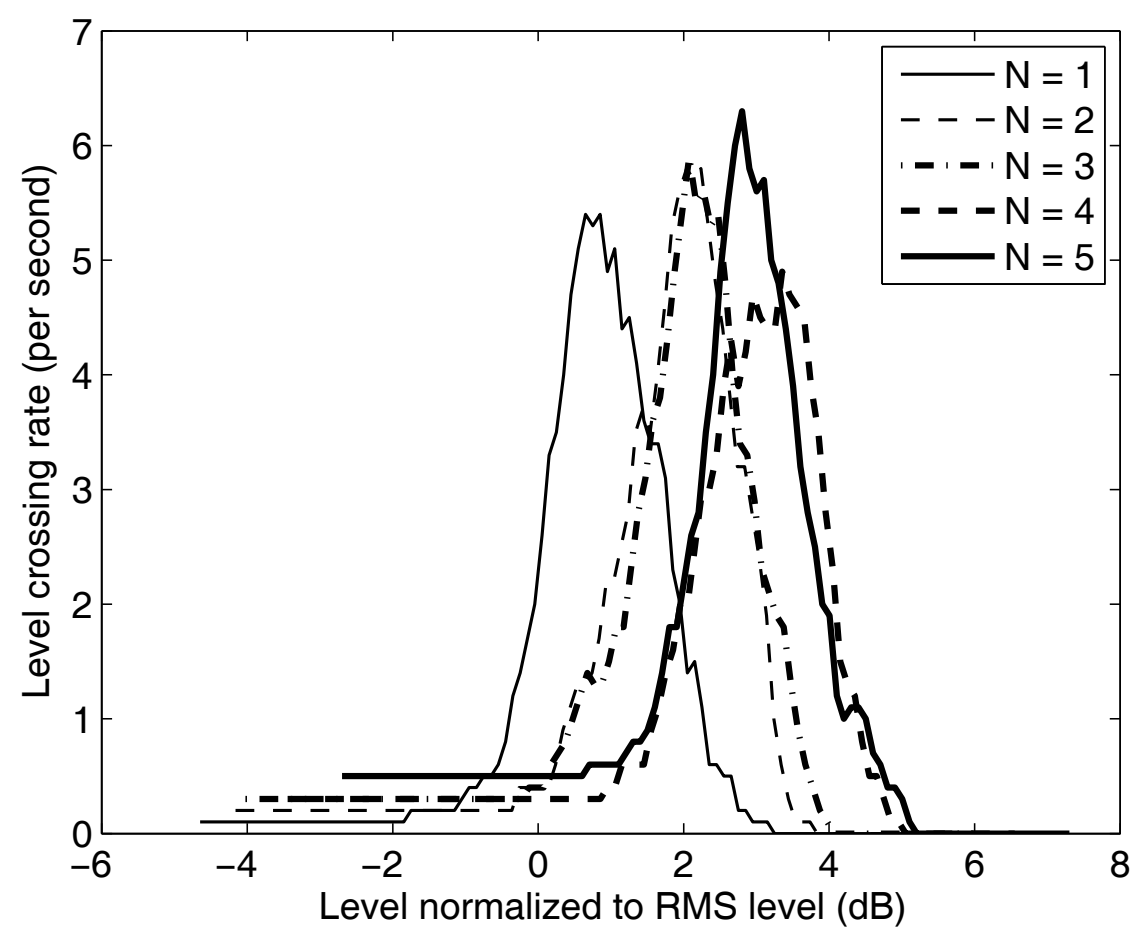

Figure 11 Simulated LCRs of the received signal effect by multiple human bodies $(N=1,2, \ldots, 5)$ at $60 \mathrm{GHz}$. The simulation parameters are given in Table 1. 
simulation time series for human body effects reported in [2] (see e.g., [2, Figure 3]) and the ones generated using the developed physical-statistical channel model shown in Figure 13. In addition, the characteristics of the simulated first and second-order statistics of the physical-statistical channel model shown in Figures 7, 8, $9,10,11$, and 12 are consistent with the ones observed from measurement results of human body effects reported in [18].

In this section, an attempt has been made to initially validate the developed physical-statistical channel model using an empirical model for human body shadowing and reported measurement results. Further validation of the proposed model will be performed in the future as radio frequency $(\mathrm{RF})$ measurements are made available.

\section{Conclusion}

A novel physical-statistical generative model for signal effect by moving human bodies is developed. The human body is modeled as vertically oriented dielectric cylindrical volume. The received signal is assumed to be composed of a direct component which might be subject to shadowing and a multipath component due to reflection and diffuse scattering, i.e., a Ricean channel. The shadowing effect of the direct signal component is calculated using Kirchhoff diffraction equation. The multipath component is parameterized by calculating the reflected fields from the floor, ceiling and walls of the indoor environment as well as scattered fields from moving human bodies. The parameters of the Rice distribution thus change as the people walk thought the propagation environment.

The shadowing and inter-shadowing events caused by multiple crossing of the LOS path are modeled using a Passion and Exponential distribution, respectively. The physical-statistical approaches discussed are more effective for simulating large scenarios compared to complex physical models, and more accurate than merely empirical or statistical models as they take into account the geometry of the link, and rely on electromagnetic based methods for calculating the needed model parameters.

Furthermore, simulation results of the first and second order statistics of the received signal affected by moving human bodies for $3.35 \mathrm{GHz}$ and $60 \mathrm{GHz}$ signals are presented. The results show that as the frequency increases the effect of moving human bodies on the first and

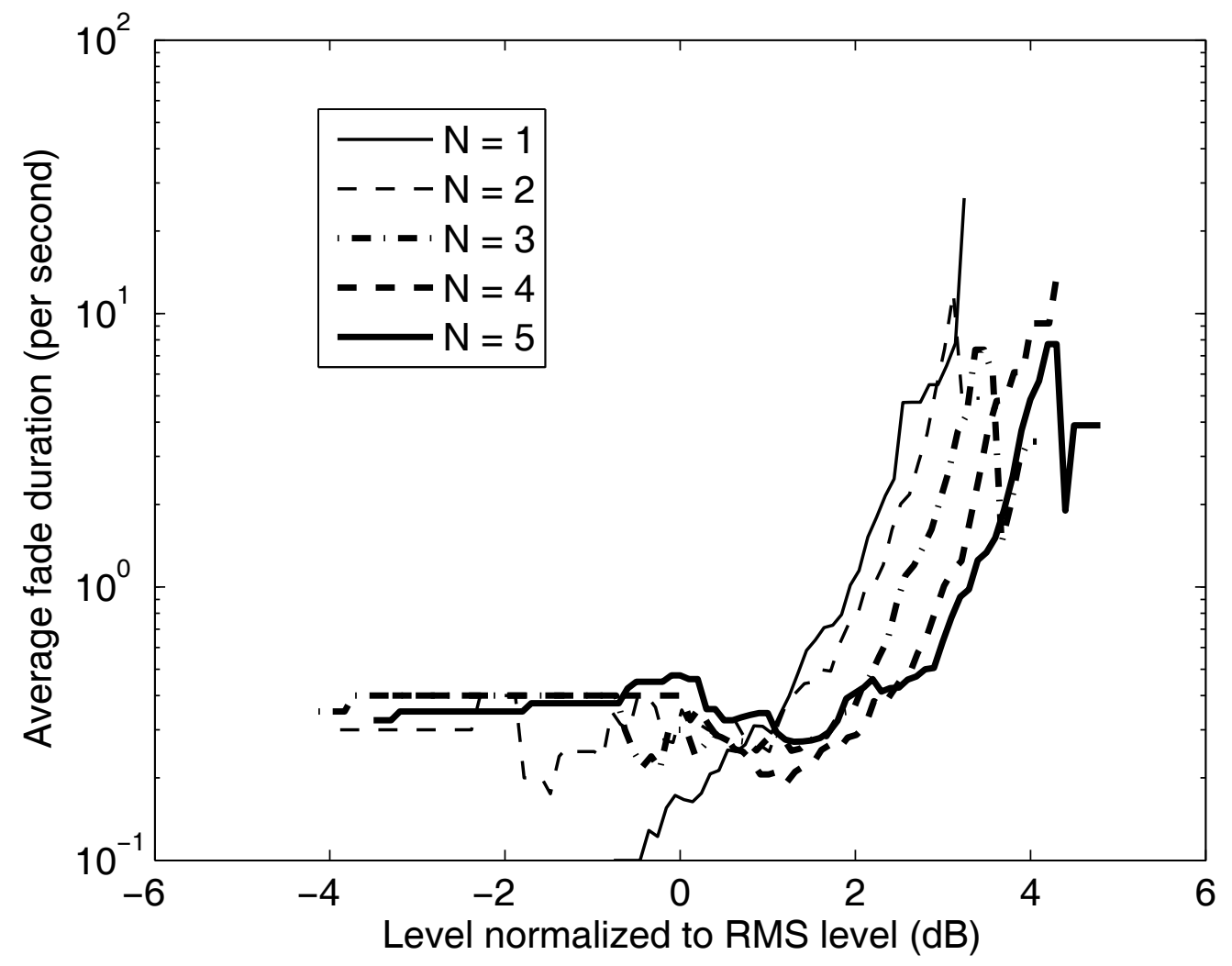

Figure 12 Simulated AFD of the received signal effect by multiple human bodies $(N=1,2, \ldots, 5)$ at $60 \mathrm{GHz}$. The simulation parameters are given in Table 1. 


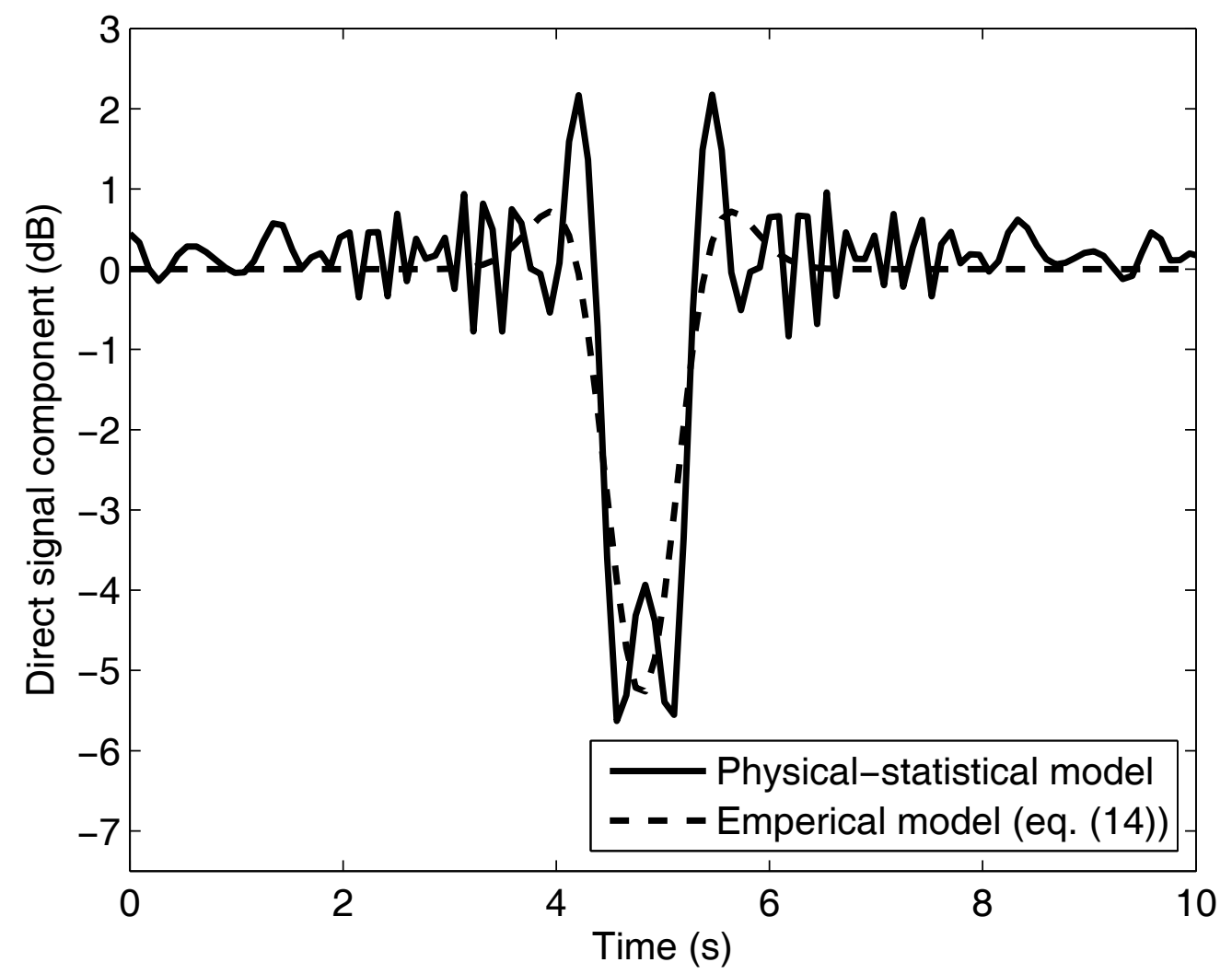

Figure 13 Comparison of the direct signal (large-scale fading) obtained using the empirical model given in Eq. (14) and the one obtained using the proposed physical-statistical model. The simulation parameters are given in Table 1 with $\widehat{N}=1, A_{s}=5.3 \mathrm{~dB}, t_{0} \mathrm{~s}, T_{s}=$ $1.2 \mathrm{~s}$ and $u=1$.

second order statistics of the channel increases. Moreover, as the number of pedestrians increase, the slope of the CDFs of the received signal decreases proportionally. Similarly, as the number of pedestrians increase, the LCRs and AFDs of the received signal increases.

An attempt has been made to initially validate the developed model using an empirical model for human body shadowing and reported measurement results. Further validation of the proposed model will be performed in the future as RF measurements are made available.

In general, the proposed model can be used to generate time series of moving human body effects for indoor wireless access systems. This enables simulation of for example capacity-enhancing techniques such as route diversity, space-time coding and adaptive coding and modulation.

\section{Acknowledgements}

The author would like to thank Gjøvik University College, Norway for supporting this study. This study was supported by Gjøvik University College, Norway.

\section{Competing interests}

The author declares that they have no competing interests.

Received: 5 December 2011 Accepted: 1 March 2012

Published: 1 March 2012

\section{References}

1. I Kashiwagi, T Taga, T Imai, Time-varying path-shadowing model for indoor populated environments. IEEE Trans Veh Tech. 59(1), 16-28 (2010)

2. M Yokota, T Ikegamai, Y Ohta, T Fujii, Numerical examination of EM wave shadowing by human body. in Proc 4th European Conf Ant Prop (EUCAP2010), Barcelona, (12-16 April, 2010)

3. AM Eid, JW Wallace, Accurate modeling of body area network channels using surface-based method of moments. IEEE Trans Ant Prop. 59(8), 3022-3030 (2011)

4. JA Robert, JR Abeysighe, A two-state Rician model for predicting indoor wireless communication performance. in Proc IEEE Int Conf Commun, Seattle, WA, 1, 40-43 (June, 1995)

5. P Pagani, P Pajusco, Chracterization and modeling of temporal variations on an ultrawideband radio link. IEEE Trans Ant Prop. 54(11), 3198-3206 (2006)

6. S Collonge, G Zaharia, GE Zein, Influence of the human activity on wideband characteristics of the $60 \mathrm{GHz}$ indoor radio channel. IEEE Trans Wirel Commun. 3(6), 2396-2406 (2004). doi:10.1109/TWC.2004.837276

7. PV Torre, L Vallozzi, L Jacobs, H Rogier, M Moeneclaey, J Verhaevert, Characterization of measured indoor off-body MIMO channels with correlated fading, correlated shadowing and constant path loss. IEEE Trans Wirel Commun. 11(2), 712-721 (2012) 
8. Propagation data and prediction methods for the planning of indoor radiocommunication systems and radio local area networks in the frequency range $900 \mathrm{MHz}$ to $100 \mathrm{GHz}$, Geneva, (2009) ITU-R P.1238-6

9. MA Karam, AK Fung, YMM Antat, Leaf-shape effects in electromagnetic wave scattering from vegetation. IEEE Trans Geosci Remote Sens. 27(6), 687-697 (1989). doi:10.1109/TGRS.1989.1398241

10. HD Hristov, Fresnel Zones in Wireless Links, Zone Plate Lenses and Antennas, Artech House, (2000)

11. FP Fontan, A Abele, B Montenegro, F Lacoste, V Fabbro, L Castanet, B Sanmartin, P Valtr, Modelling of the land mobile satellite channel using a virtual city approach. in Proc 2nd European Conf Ant Prop (EUCAP2007), Edinburgh, (11-16 November, 2007)

12. RH Clarke, A statistical theory of mobile radio reception. Bell System Tech J. 47, 957-1000 (1968)

13. RJC Bultitude, Measurement characterization and modeling of indoor 800/ $900 \mathrm{MHz}$ radio channels for digital communications. IEEE Commun Meg. 25(6), (5-12 June1987)

14. SJ Howard, K Pahlavan, Doppler spread measurements of indoor radio channel. Electron Lett. 26(2), 107-109 (1990). doi:10.1049/el:19900074

15. JB Andersen, JO Nielsen, GF Pedersen, G Bauch, Doppler spectrum from moving scatterers in a random environment. IEEE Trans Wirel Commun. 8(6), 3270-3276 (2009)

16. DS Promislow, Poisson Processes, in Fundamentals of Actuarial Mathematics, 2nd edn. John Wiley and Sons Ltd., Chichester, UK, (2010)

17. LLC-Books, Poisson Processes, Exponential Distribution, Shot Noise, Poisson Process, Radioactive Decay, Poisson Distribution. Proportional Hazards M. Books LLC, Wiki Series, (2010) ISBN:978-1155717906

18. KI Ziri-Castro, NE Evans, WG Scanlon, Propagation modelling and measurments in a populated indoor environment at $5.2 \mathrm{GHz}$. Auswirel Conf, Sydney, (13-16 March, 2006)

19. CC Johanson, AW Guy, Nonionizing electromagnetic wave effects in biological materials and systems, in Proc IEEE. 60(6), 692-718 (1972)

20. N Chahat, M Zhadobov, R Augustine, R Sauleau, Human skin permittivity models for millimetre-wave range. Electron Lett. 47(7), 427-428 (2011). doi:10.1049/el.2011.0349

doi:10.1186/1687-1499-2012-77

Cite this article as: Cheffena: Physical-statistical channel model for signal effect by moving human bodies. EURASIP Journal on Wireless Communications and Networking 2012 2012:77.

\section{Submit your manuscript to a SpringerOpen ${ }^{\circ}$ journal and benefit from:}

- Convenient online submission

- Rigorous peer review

- Immediate publication on acceptance

- Open access: articles freely available online

- High visibility within the field

- Retaining the copyright to your article

Submit your next manuscript at $\gg$ springeropen.com 